\title{
Detection of bacteraemia by the continuously monitoring BacT/Alert system
}

\author{
G T Kennedy, J G Barr, C Goldsmith
}

\begin{abstract}
Aims-To analyse a continuously monitoring blood culture system with respect to the time to detection of various groups of organisms, their clinical importance, and the relative efficacy of the aerobic and anaerobic bottles.

Methods-Four thousand blood cultures were monitored and the information relating to the positive cultures was noted and analysed.

Results-Four hundred and seventy seven blood cultures were detected as positive, $81 \%(387 / 477)$ of which were detected within 48 hours. The most pathogenic organisms were detected in the shortest period, less pathogenic later and those generally regarded as contaminants last. Clinically important isolates were also detected earlier. Many positive blood cultures were detected in only one bottle of the set, even those regarded as clinically important.

Conclusions-The management of continuously monitoring blood culture systems could be improved by considering time to detection trends. Clinicians should be aware of the relatively rapid detection of clinically important, positive blood cultures in relation to patient treatment.

( $\mathcal{~ C l i n ~ P a t h o l ~ 1 9 9 5 ; 4 5 : 9 1 2 - 9 1 4 ) ~}$
\end{abstract}

Keywords: BacT/Alert, continuous monitoring, time to detection trends.

The detection of bacteraemia and septicaemia remains one of the most important services provided by clinical bacteriology laboratories. ${ }^{12}$ Management of infection in many cases is commenced on an empirical basis: this is especially the case in immunocompromised or elderly patients who may not present with the usual clinical signs and symptoms. It is therefore important to be able to detect bacteraemia/ septicaemia as early as possible and to identify the causative pathogens and determine antibiotic sensitivity, so that optimal patient management can be instituted as early as possible.

The methods of detecting positive blood cultures has changed progressively from manual subculture and the examination of plates to semi-automated batch detection, particularly using the BACTEC radiometric systems. The past few years have seen the development and marketing of a new generation of blood culturing machines which utilise continuously monitoring technology. These systems have included machines which detect positive blood cultures by colorimetric $\mathrm{CO}_{2}$ sensors (BAC-
TEC 9240: Becton Dickinson Diagnostic Instrument Systems, Sparks, Maryland, USA) and by relative changes in voltage produced by bacterial growth in the culture media (Sentinel: Difco Laboratories Ltd, East Molesey, Surrey, UK). Comparative studies between continuously monitoring and batch monitoring blood culture systems have been undertaken with the Sentinel and BacT/Alert systems: these studies indicated that the continuously monitoring systems were comparable for the recovery of clinically important microorganisms. ${ }^{34}$

In this large teaching hospital, 10 years of experience with a radiometric BACTEC system for blood cultures has been followed in the past year by use of the continuously monitoring BacT/Alert. This study reports on the spectrum of organisms recovered over the study period, their time to detection, the clinical importance of the isolate, and the contribution made by the aerobic and anaerobic blood culture bottles.

\section{Methods}

For the duration of the study, blood cultures, which were submitted to the laboratory for routine analysis, were loaded onto the BacT/ Alert system (Organon Teknika, Durham, North Carolina, USA) and monitored for the presence of micro-organisms. Each set consisted of an aerobic and an anaerobic bottle each inoculated with 5-10 ml of patient blood. The aerobic BacT/Alert blood culture bottle contained $40 \mathrm{ml}$ of media comprising pancreatic digest of casein $(1.95 \% \mathrm{w} / \mathrm{v})$, papain digest of soybean meal $(0.3 \% \mathrm{w} / \mathrm{v})$, sodium polyanetholsulphate $(0.035 \% \mathrm{w} / \mathrm{v})$, pyridoxine $\mathrm{HCl}(0.001 \% \mathrm{w} / \mathrm{v})$, and complex amino acid and carbohydrate substrates in purified water. Bottles were prepared with an atmosphere of $\mathrm{CO}_{2}$ in air under vacuum. The anaerobic bottles also contained menadione $(0.00005 \%$ $\mathrm{w} / \mathrm{v})$, hemin $(0.0005 \% \mathrm{w} / \mathrm{v})$, reducing agents, and $\mathrm{CO}_{2}$ in nitrogen under vacuum media was supplied by Organon Teknika.

The BacT/Alert system consists of at least one cabinet for incubating, agitating and monitoring up to 240 blood culture bottles and a personal IBM compatible computer, which contains software that controls all aspects of the continuously monitoring process. The BacT/ Alert system operates by spectrophotometric detection of $\mathrm{CO}_{2}$. Organisms, if present, produce $\mathrm{CO}_{2}$ which diffuses across an ion impermeable membrane at the base of the bottle, where it comes into contact with a $\mathrm{pH}$ indicator. The indicator is saturated with water; hydrogen ions are generated, and this progressive change 
Isolates from blood cultures and their frequency

\begin{tabular}{lrlr}
\hline Micro-organism & $n$ & Micro-organism & $n$ \\
\hline Acinetobacter calcoaceticus & 3 & Proteus vulgaris & 5 \\
Bacillus spp & 1 & Pseudomonas maltiphilia & 4 \\
Bactereroides fragilis & 4 & Pseudomonas spp & 1 \\
Candida parapsilosis & 8 & Serratia liquefaciens & 5 \\
Citrobacter freundii & 1 & Staphylococcus aureus & 50 \\
Coliform spp & 7 & Staphylococcus (coagulase negative) & 181 \\
Corynebacterium JK & 1 & Streptococcus acidimonas & 2 \\
Diptheroids & 27 & Streptococcus agalactiae & 4 \\
Enterobacter aerogenes & 4 & Streptoccus cremoris & 2 \\
Enterobacter agglomerans & 10 & Streptococcus milleri & 3 \\
Enterobacter cloacae & 7 & Streptococcus mitis & 1 \\
Enterococus faecalis & 13 & Streptococcus morbillorum & 2 \\
Enterococcus faecium & 3 & Streptococcus pneumonia & 10 \\
Escherichia coli & 42 & Streptococcus pyogenes & 2 \\
Haemophilus influenzae & 3 & Streptococcus sanguis & 6 \\
Morganella morganii & 3 & Streptococcus spp & 5 \\
Niesseria flavescens & 1 & Veillonella spp & 2 \\
Nieisseria meningitidis & 5 & Yeast & 1 \\
Propionibacterium & 6 & Mixed & 25 \\
Proteus mirabilis & 2 & No growth & 15 \\
\hline & & &
\end{tabular}

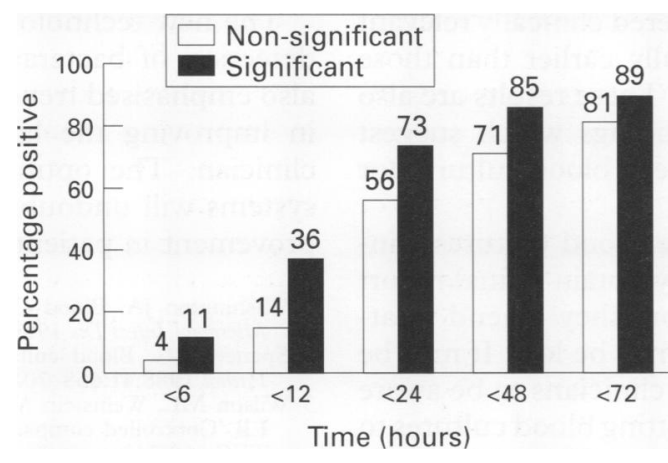

Figure 1 Time to detection of all positive blood cultures.

in $\mathrm{pH}$ causes the colour to change from a dark green through to a bright yellow, the intensity of which depends on the quantity of $\mathrm{CO}_{2}$ present. This colour change is monitored by a sensor located at the base of the receptive silo on the machine every 10 minutes. An algorithm in the computer is used to decide the status of the bottle and is dependent on three parameters: the initial value of $\mathrm{CO}_{2}$, the rate of $\mathrm{CO}_{2}$ production and the increase in the rate of $\mathrm{CO}_{2}$ production. A positive culture is indicated by an audible alarm.

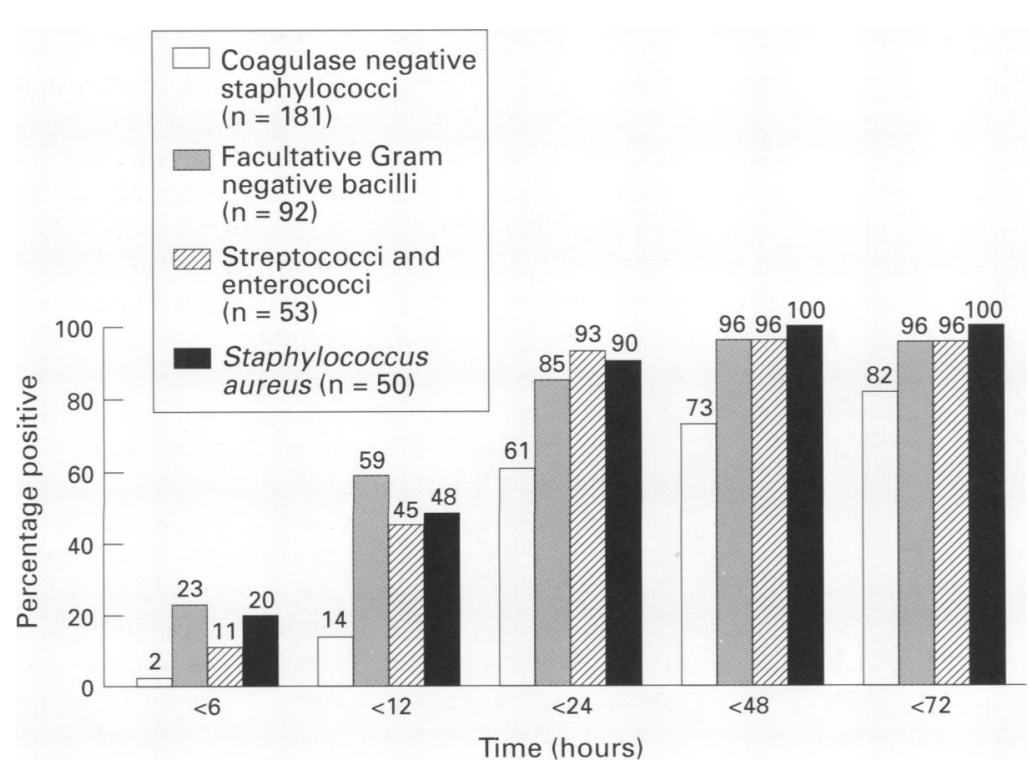

Figure 2 Time to detection for selected groups of micro-organisms.
Positive cultures were then removed from the system and approximately $1 \mathrm{ml}$ of the blood/ broth mixture was removed and Gram stained. The mixture was then inoculated onto Colombian blood agar, chocolate agar, MacConkey agar, and neomycin blood agar. Identification was carried out the following day using standard materials as appropriate: these included Gram stain, catalase test, tube coagulase tests API 20E, API 20NE and API STREPT.

The time taken for detection was noted. The significance of the blood cultures was assessed as described by Glenister et al. ${ }^{5}$ Blood cultures were regarded as significant if the patient had one of the following: (1) fever (greater than or equal to $37 \cdot 8^{\circ} \mathrm{C}$ ); (2) hypothermia (less than or equal to $36^{\circ} \mathrm{C}$ ); (3) rigor; or (4) hypotension. In addition, the patient should have one of the following: (1) increased white cell count, raised erythrocyte sedimentation rate, or raised $C$ reactive protein concentration; (2) clinical evidence of cardinal symptoms of infection (according to Glenister); or (3) abolition of any of the above indications of infection in response to appropriate antibiotic therapy, incision, drainage or removal of a venous catheter.

\section{Results}

Four thousand bottles were analysed during the study, of which 477 were positive. This represented 313 sets. Of these, 222 were regarded as significant, and 91 as not significant. Isolates and their frequencies are shown in the table.

Twenty nine percent of all positive blood cultures were detected in less than 12 hours, $68 \%$ by 24 hours, $81 \%$ by 48 hours, and $87 \%$ by 72 hours. The remaining $13 \%$ were detected within seven days.

The time to detection for significant and non-significant blood cultures is shown in fig 1. At 12 hours, $45 \%$ of the streptococci and enterococci were detected, $48 \%$ of Staphylococcus aureus infections and $59 \%$ of the facultative Gram negative species. Fourteen percent of the coagulase negative staphylococci and $18 \%$ of the diptheroids and propionibacteria were detected within 12 hours. The time to detection for specific groups of organisms is shown in fig 2 .

Of the 313 sets of blood cultures, 157 (50\%) were positive in both aerobic and anaerobic bottles. In $106(68 \%)$ of 157 cases the aerobic bottle became positive before the anaerobic bottle. Of those considered to be significant (222 sets), 125 (56\%) were detected in both bottles. Eighty eight $(70 \%)$ of these were detected by the aerobic bottle first.

\section{Discussion}

The isolation rate of organisms in the present study $(11.6 \%)$ was similar, but not significantly different, from that obtained using the BACTEC 460 in two previous equivalent periods in 1992 and 1993 (9.7\% and 9.9\%). The spectrum of isolates obtained was similar in all three years and was considered representative of the 
types of organisms commonly isolated from blood cultures.

Continuous monitoring demonstrates that most positives are detected in a relatively short period of time, the majority within 24 hours. There also appears to be a trend for organisms in this laboratory which are considered most pathogenic to be detected in the earliest stages of monitoring (Staphylococcus aureus, Gram negative bacilli, streptococci, and enterococci). The vast majority of these are detected within 24 hours. This is followed by those of more debatable pathogenicity (coagulase negative staphylococci), which take considerably longer. Finally, those generally regarded as contaminants (diptheroids and propionibacteria) are detected much later than other isolates.

This trend is emphasised in this study in that those isolates considered clinically relevant were detected substantially earlier than those regarded as not relevant. These results are also consistent with other findings which suggest that there is no need to keep blood cultures for more than five days. ${ }^{6}$

In the case of negative blood cultures clinicians may wait until they obtain a final report from the laboratory before they amend treatment and valuable time may be lost. It may be considered of benefit for clinicians to be aware of these facts when submitting blood cultures to the microbiology laboratory. It is also suggested that the common practice of issuing negative reports after seven days may be misleading and it may be helpful to issue an intermediate report after 48 or even 24 hours. Such practices may be beneficial by reducing antibiotic misuse. Certainly, every effort should be made to inform clinicians of the percentage of significant blood cultures becoming positive in 24 or 48 hours. It should also be insured that those responsible for determining treatment are aware of the laboratory's reporting procedure.

A large proportion of blood cultures were positive in only one of the bottles and those regarded as significant were only slightly more likely to be found in both aerobic and anaerobic bottles. This may be because the very low numbers of organisms present in the initial inoculum and/or the blood not being equally distributed over the two bottles. The importance of the aerobic bottle has once again been demonstrated. Too much emphasis may therefore be placed on the view that only significant blood cultures will be found in both bottles when no attempt is made to quantitate the blood cultured in each bottle.

The new technology has not only made the detection of bacteraemia more rapid but has also emphasised trends which could be utilised in improving the laboratory's service to the clinician. The optimal management of such systems will undoubtedly offer significant improvement in patient care.

1 Washington JA. Blood Cultures: An overview. Eur $f$ Clin Microbiol Infect Dis 1989;8:803-6.

2 Spencer RC. Blood cultures: where do we stand? 7 Clin Pathol 1988;41:668-70.

3 Wilson ML, Weinstein MP, Reimer LG, Mirrett S, Reller LB. Controlled comparison of the BacT/Alert and BACTEC 660/730 nonradiometric blood culture systems. F Clin Microbiol 1992;30:323-9.

4 Stevens M, Patel H, Walters A, Burch K, Jay A, Dowling N, et al. Comparison of Sentinel and BACTEC blood culture systems. $\mathcal{F}$ Clin Pathol 1992;45:815-18.

5 Glenister HM, Taylor LJ, Cooke EN. Study of surveillance methods for detecting hospital infection. London: PHLS Central Public Health Laboratory, 1992.

6 Thorpe TC, Wilson ML, Turner JE, DiGuiseppi JL, Willert M, Mirrett S, et al. BacT/Alert: an automated colorimetric microbial detection system. F Clin Microbiol 1990;28:160812 . 